\title{
Placing Edge Labels by Modifying an Orthogonal Graph Drawing
}

\author{
Konstantinos G. Kakoulis ${ }^{1}$ and Ioannis G. Tollis ${ }^{2}$ \\ 1 Dept. of Industrial Design Engineering, \\ T.E.I. of West Macedonia, Greece \\ kkakoulis@teikoz.gr \\ 2 Dept. of Computer Science, University of Crete and \\ FORTH-ICS, Crete, Greece \\ tollis@ics.forth.gr
}

\begin{abstract}
In this paper we investigate how one can modify an orthogonal graph drawing to accommodate the placement of overlap-free labels with the minimum cost. We present a polynomial time algorithm that finds the minimum increase of space in one direction, needed to resolve overlaps, while preserving the orthogonal representation of the drawing.
\end{abstract}

\section{Introduction}

Automatic labeling is a very difficult problem, and because we rely on heuristics to solve it, there are cases where the best methods available do not always produce an acceptable or legible solution even if one exists. If a solution to the labelng problem is not acceptable one could either redraw the graph taking into account the placement of labels (in 112/3 labeling with drawing of orthogonal representations of graphs is combined), or modify the drawing to produce an acceptable label assignment.

In this paper we consider the problem of modifying an existing orthogonal drawing by inserting extra space in order to accommodate the placement of edge labels that are free of overlaps. We will refer to it as the Opening Space Label Placement (OSLP) problem. We have chosen orthogonal drawings because they have the most regular structure among all layout styles with respect to opening space, which in orthogonal drawings implies inserting rows and/or columns.

\section{Solving the OSLP Problem}

We want to minimize the extra area needed to resolve label overlaps while preserving the orthogonal representation of the drawing. Because the OSLP problem is NP-Complete [4] we must rely on heuristics to solve it.

Given an orthogonal drawing $\Gamma$, first we find an edge label assignment where overlaps are allowed by using existing techniques. Then, we modify $\Gamma$ by applying a polynomial time algorithm based on minimum flow techniques to find the minimum width needed to eliminate label overlaps in $\Gamma$, given a partial order of overlapping objects, while preserving the orthogonal representation of $\Gamma$. The case of finding minimum height is analogous. 

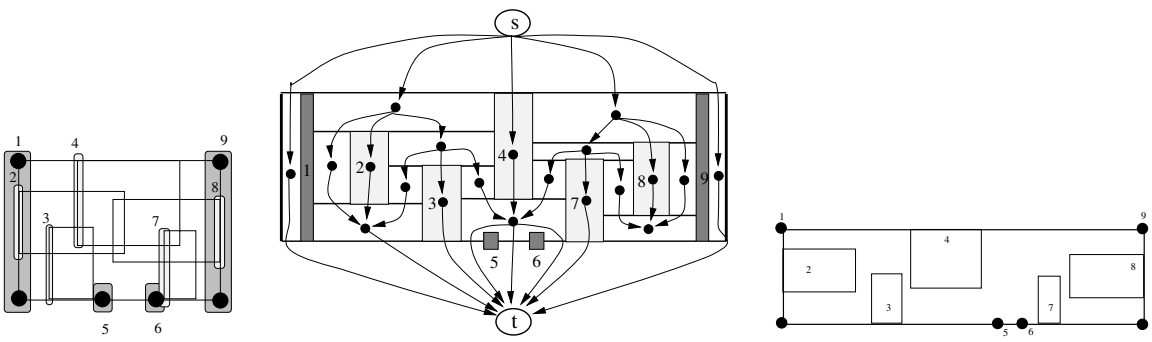

Fig. 1. (a) An input drawing $\Gamma$ with label overlaps. (b) The flow graph for $\Gamma$. (c) Label overlaps have been resolved by running the minimum flow algorithm.

The main idea of our algorithm is the following: We create a directed acyclic graph $G_{\text {flow }}$ that transfers flow from the top to the bottom of the drawing in order to insert extra vertical space to resolve horizontal overlaps. Intuitively, if two objects overlap, then we must push between them at least as much flow as the amount of their overlap in the $x$ direction. First we decompose the input drawing $\Gamma$ into vertical segments (each label, node and vertical edge segment of $\Gamma$ is a vertical segment). Next, we obtain the partial order of the objects by performing a plane sweep. Then, we create a separation visibility graph $G_{s v}$. For each vertical segment of $\Gamma$ we insert a node in $G_{s v}$. Each node in $G_{s v}$ is a rectangle and has the size of its corresponding object in $\Gamma$. We insert edges in $G_{s v}$ by expanding (to the left and to the right) the horizontal sides of each node in $G_{s v}$ until they touch another node of $G_{s v}$. We assign a weight to each edge $e$ of $G_{s v}$ which represents the minimum distance the two objects connected with edge $e$ must be kept apart to avoid overlaps. Next, we create the flow graph $G_{\text {flow }}$ from graph $G_{s v}$ by adding into $G_{\text {flow }}$ : $(i)$ A source node $s$ and a target node $t,(i i)$ A node for each face of $G_{s v}$ and (iii) An edge for each pair of neighboring faces of $G_{s v}$ that share a horizontal edge segment. For each edge in $G_{\text {flow }}$ we assign lower and upper capacity equal to a pair of weights for the only edge in $G_{s v}$ it intersects. We show that the minimum flow of $G_{f l o w}$ produces the minimum width expansion needed to resolve overlaps.

Theorem 1. The minimum flow of $G_{\text {flow }}$ gives the minimum width of the drawing, in $O(m \log n(m+n \log n))$ time, such that all label overlaps are resolved in one direction and the orthogonal representation of the drawing is preserved.

\section{References}

1. Binucci, C., Didimo, W., Liotta, G., Nonato, M.: Orthogonal Drawings of Graphs with Vertex and Edge Labels. CGTA 32(2), 71-114 (2005)

2. Di Battista, G., Didimo, W., Patrignani, M., Pizzonia, M.: Orthogonal and quasiupward drawings with vertices of arbitrary size. In: Kratochvíl, J. (ed.) GD 1999. LNCS, vol. 1731, pp. 27-37. Springer, Heidelberg (1999)

3. Klau, G.W., Mutzel, P.: Combining Graph Labeling and Compaction. In: Kratochvíl, J. (ed.) GD 1999. LNCS, vol. 1731, pp. 27-37. Springer, Heidelberg (1999)

4. Kakoulis, K.G., Tollis, I.G.: On the Complexity of the Edge Label Placement Problem. Computational Geometry 18(1), 1-17 (2001) 